\title{
Transitory Thrombocytopenia in Newborn Mice after Intrauterine Hypoxia
}

\author{
AI.1 MI:BI:R(; $i^{1+1}$

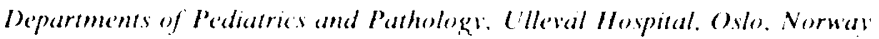

\begin{abstract}
Summary
Pregnant mice were exposed to hypobaric hypoxia $(0.7$ atmospheres) continuously during days 13 to 20 of pregnancy. In the newborn offspring, the body weights were decreased, and liver weight/hody weight ratios were increased compared to nonhypoxic control animals $(P<0.001)$. After intrauterine hypoxia, the newborn animals were polycythemic, and a postnatal transitory thrombocytopenia lasting 7 days occurred. A rebound thrombocytosis appeared on days 13 to 15 after birth. Bone marrow megacaryocyte concentrations were increased in the hypoxic animals on days 5 to 15 after birth compared to the nonhypoxic controls. These findings may be explained by a competitive mechanism on common stem cells. Increased demand for erythropoiesis during hypoxia may shunt hematopoietic cells in direction of erythropoiesis at expense of thrombopoiesis.
\end{abstract}

\section{Speculation}

Common stem cells for erythropoiesis and megacaryopoiesis may exist. Increased demand for erythropoiesis during long-term hypoxia may shunt hematopoietic cells in direction of erythropoiesis at expense of thrombopoiesis. Placental dysfunction may impair oxygenation of the fetus. explaining the frequent occurring polycythemia and transitory postnatal thrombocytopenia in growth-retarded newborn human infants.

Hypoxic exposure has been shown to cause a decrease of the platelet count in adult mice (11) and rats (5). This seems to be calused by a decreased platelet production (5). In human infants with intraterine growth retardation. transitory postnatal thrombocytopenia is a frequent tinding $(x)$. These infants may have developed under a chronic hypoxic condition in utero because of placental dysfunction. The well-known polycythemia found in these infants supports this view (1.4.8).

The present study was performed to answer the following question: can the syndrome of growth retardation. polycythemia, and transitory postnatal thrombocytopenia be caused by longterm fetal hypoxia?. The pregnant mouse was chosen as an experimental model. Body and liver weights vere registered in new born mice at birth. and hematocrit kevels. thromberyle counts, and bone marrow megacaryocyte concentrations were registered during the first 15 days of life in mice exposed to chronic intratuterine hypoxia and in nonhypoxic control animals.

\section{MATLRIALS AND METHODS}

Virgin WI.O-mice $\&$ to $10 \mathrm{wk}$ old were matted to mate mice of the same strain. From the 13 th until the 20 th day after mating. pregnant mice were exposed to hypobaric hypoxia of 0.7 atmospheres. Hypoxia was given continuously except for opening the hypoxia chamber twice daily for 10 min for cleaning and supplies. On day 20. the mice were transferred to eages, and the pups which were born within $24 \mathrm{hr}$ after taking the mothers out of the chamber were included in the investigation. Mice born in the hypoxia chamber were excluded on account of the possible effects of extrauterine hypoxia. Nonhypoxic animals of corresponding age were used as controls.

The mated female mice were investigated for body weights before mating and on the day after delivery. In newborn mice from litters consisting of 7 or 8 pups, body and liver weights were registered on the day of birth. Body weights were registered using an electronic balance (Mettler PL 1200); Mettler Instrumente Ab. Zurich). Livers were taken out in extenso using a binocular dissecting microscope (Zeiss, Göttingen. West Germany). Liver weights were measured (Type HI6 balance: E. Mettler. Zürich.

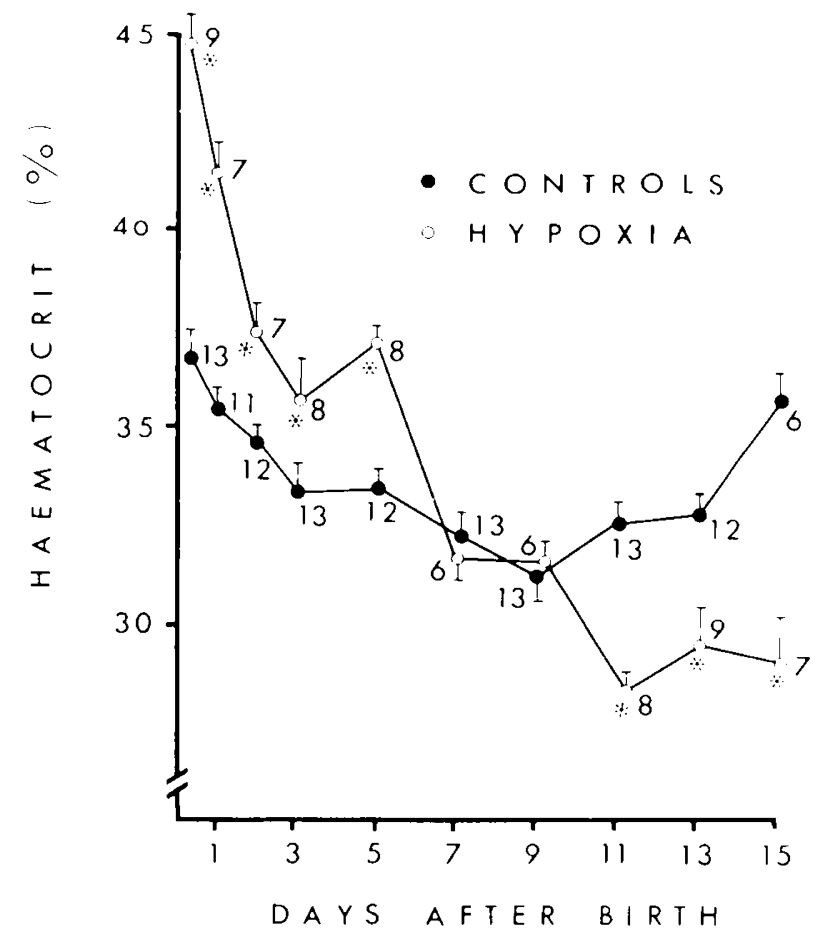

Fig. 1. Hematocrit levels $\pm \$ .1$ (har) on the tirst 15 dats at lite in mice after intrauterine hypoxic exposure (0.7 atmospheres for 8 days) and in normal nonhypoxic mice. ${ }^{*}$. statistical signiticant difference $(P<0.05)$ between the two groups. Numerals. number of observations for each point.

Switzerland) and related to the body weight of the corresponding mouse.

The hemalocrit levels. platelet counts. and bone marrow megacaryocyle concentrations were registered during the first 15 days of life in randomly selected animals. Blood samples were taken after transecting the cervical hlood vessels. The hematocrit was measured by a routine microhematocrit method. and platelet counts were measured by phase contrast microscopy. Bone marrow megacaryocyte concentrations were investigated on $5-\mu \mathrm{m}$ longitudinal sections of femoral marrow tixed in Zenker-formol solu- 
Table 1. Body, weights, liver weights, and liver weight/body weight ratios on the first day of life in normal mice and in offsprings of mice exposed to hypobaric hypoxia $(0.7$ atmospheres) on dats 1.3 to 20 of pregnancy

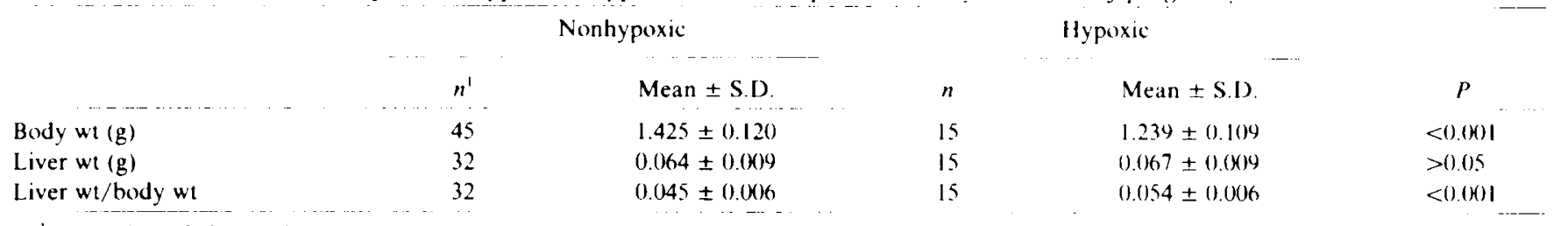

' n, number of observations.

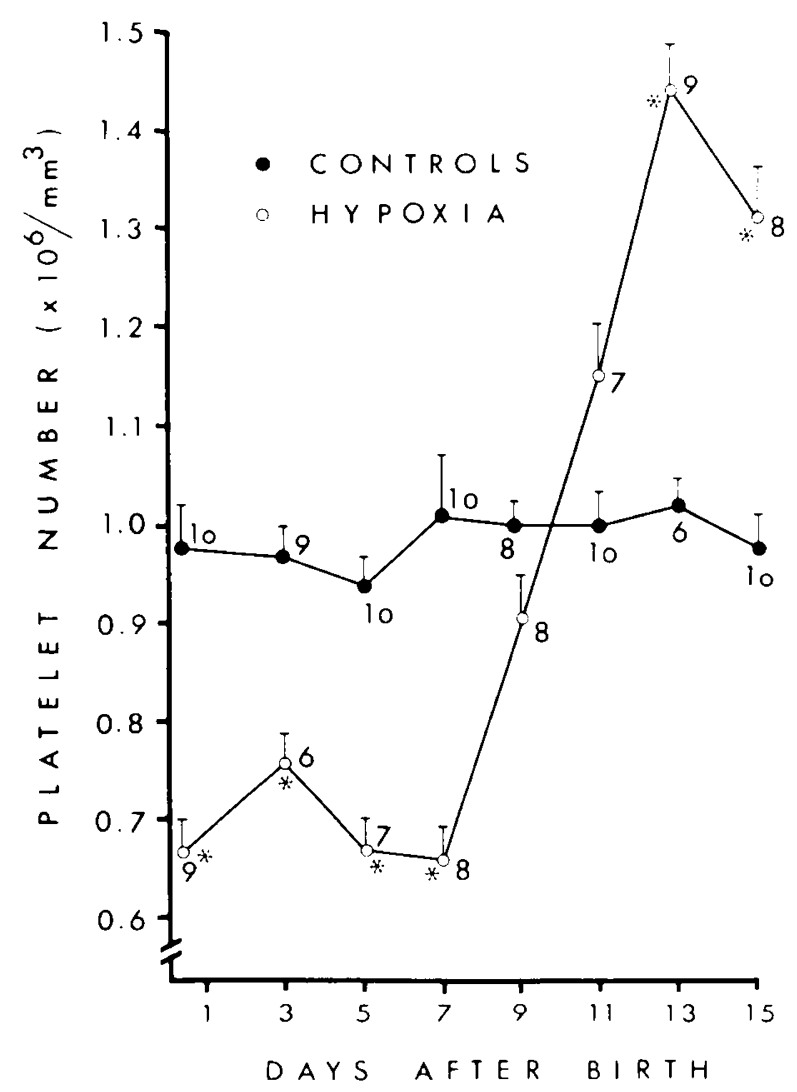

Fig. 2. Platelet counts \pm S.E. (hars) on the tirst 15 days of life in mice after intrauterine hypoxic exposure ( 0.7 atmospheres for 8 days) and in normal nonhypoxic mice. ${ }^{*}$. statistical significant difference between the two groups $(P<0.05)$. Numerals, number of observations for each point.

tion, decalcified, and stained by Wright-Giemsa. The average number of megacaryocytes per high power field (magnification. $\times 40$ ) was calculated from three longitudinal sections for each animal. The sections were coded before counting.

Differences in body weights, liver weights, liver weight/bodyweight ratios, and bone marrow megacaryocyte concentrations between the hypoxic and nonhypoxic groups were tested by means of the Student's $t$ test. Differences in hematocrit levels and thrombocyte counts were tested for by means of Wilcoxon's rank sum test.

\section{RESULTS}

Body weights of the female mice (mean \pm S.D.) before mating were $23.6 \pm 2.3 \mathrm{~g}(n=33)$ and $23.2 \pm 2.1 \mathrm{~g}(n=35)$. respectively. for the control group and the hypoxic group $(P>0.05)$, whereas the body weights on the day after delivery were $30.8 \pm 2.3 \mathrm{~g}$ ( $n$ $=31)$ and $27.9 \pm 2.7 \mathrm{~g}(n=32)$, respectively $(P<0.001)$.

Table 1 shows the body weights, liver weights, and liver weight/ body weight ratios on the first day of life in the nonhypoxic and hypoxic animals. The hypoxic mice had signiticantly lower body weights and higher liver weight/body weight ratios than did the nonhypoxic control group $(P<0.001)$.

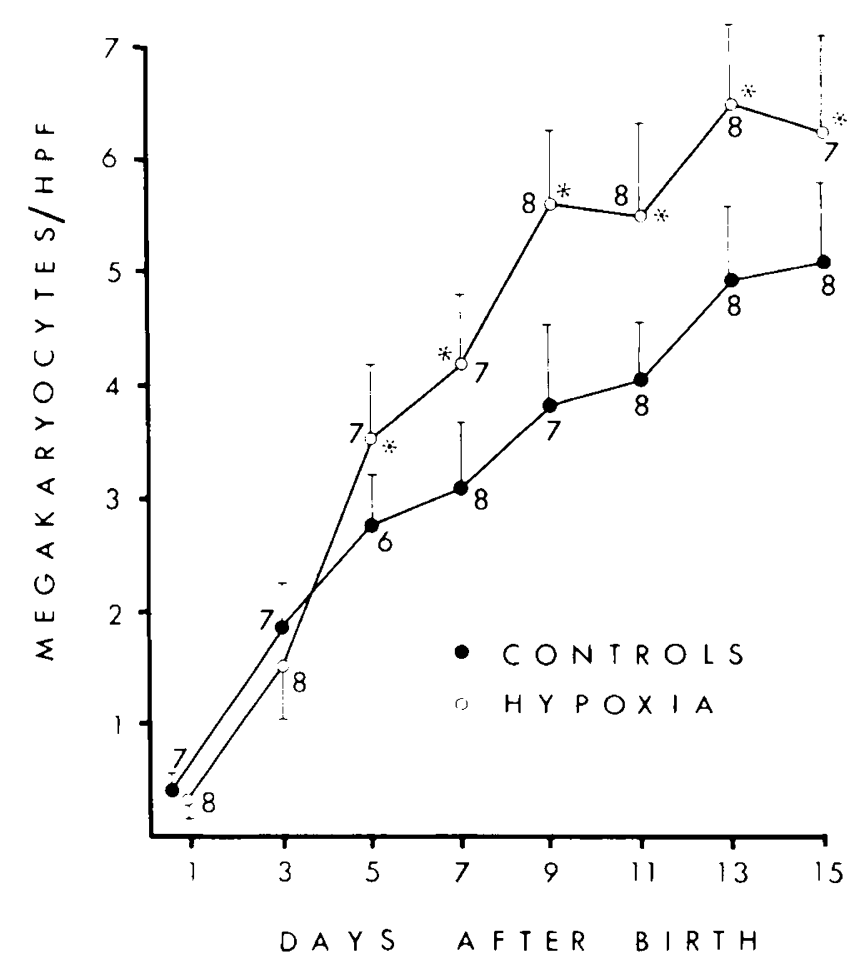

Fig. 3. Megacaryocyte counts per high power field ( $/ P P$ : magnificatoon. $\times 40) \pm S . D$. in temoral bone marrow on the first is days of lote in mice after intrauterine hypoxic exposure $(0.7$ atmospheres for 8 days $)$ and in normal nonhypoxic mice. ${ }^{*}$. statistical significant difference $(P<0.05)$ between the two groups. Numerals. number of animals investigated for each point.

Figure 1 shows the hematocrit levels during the first 15 days of life in the two groups. The hypoxic newborn animals had significantly higher hematocrit levels on the first 5 days after birth and significantly lower levels on days 11 to 15 compared to the control group $(P<0.05)$.

Figure 2 shows the thrombocyte counts on the first 15 days of life in the two groups. The hypoxic animals had significantly decreased levels during the first 7 days of life and elevated levels on days 13 to 15 compared to the control group $(P<0.05)$.

Figure 3 shows the megacaryocyte concentrations in femoral bone marrow during the first 15 days of life in the two groups. The numbers increased during the observation period in both groups. Higher megacaryocyte numbers were found on days 5 to 15 after birth in the hypoxic animals than in the control group $(P$ $<0.05)$.

\section{DISCUSSION}

In the present investigation, growth retardation, polycythemia. and a transitory postnatal thrombocytopenia was found in newborn mice after chronic intrauterine hypoxia. Impaired oxygenation of the fetus may interfere with intrauterine growth. causing a reduction in body weight at birth. This is consistent with the general growth retardation found in human infants born after domicile at high altitude compared to infants born near sea level 
(6). It cannot be excluded. however. that the lower weight gain of the pregnant mice exposed to hypoxia compared to the nonhypoxic control animals reflects a lower food intake in these animals. This may have contributed to the growth retardation of their newborn offspring.

Hypoxia stimulates erythropoiesis through increased erythropoietin production. This occurs in fetal mice and rats as well as in adult animals (7). In the fetal and newborn mouse, the liver is the main erythropoietic organ (9). The increased liver weight/body weight ratio found in hypoxic newhorn animals compared to the nonhypoxic animals (Table 1) points to a stimulated erythropoiesis in the liver during intrauterine hypoxia. This is further supported by the elevated hematocrit levels found after birth in the hypoxic mice (Fig. 1).

The platelet counts in the hypoxic newborn mice were found decreased to 60 to $70 \%$ of the values of the nonhypoxic control group during the first wk of life (Fig. 2). These findings are in agreement with the results obtained in adult mice $(11)$ and rats (5) after prolonged hypoxic exposure. The thrombocytopenic phase was in the present study followed by a rebound thrombocytosis on days 13 to 15 after birth (Fig. 2). The inverse relationship between the hematocrit levels and thrombocyte counts found in newborn mice after chronic intrauterine hypoxia (Figs. 1 and 2) may be explained by a competitive mechanism on common stem cells for erythropoiesis and thrombopoiesis. Increased demand for erythropoiesis during long-term hypoxia may shunt hematopoietic cells in direction of erythropoiesis at expense of thrombopoiesis. This view is supported by the study of Jackson and Edwards (5) who found a decreased megacaryocyte concentration in bone marrow in adult rats during hypoxic exposure. followed by an elevated concentration after termination of hypoxia. Although the platelet count was reduced, the megacaryocytes were stimulated. suggesting that the thrombocytopenia did not result from a general inhibition of thrombopoiesis, but rather a specific inhibition of precursor differentiation. Similar interdependency between erythropoiesis and thrombopoiesis has been found in adult mice by Cooper and Cooper (2).

In the mouse. hepatic erythropoiesis disappears during the first one to $2 \mathrm{wk}$ after birth (9), coincident with a rapidly increasing bone marrow hematopoiesis. This is also reflected by the increasing megacaryocyte concentrations in bone marrow after birth (Fig 3 ). In the hypoxic animals. there may be an increased influx of precursor cells for thrombopoiesis during the thrombopenic phase after birth. explaining the increased megacaryocyte concentrations in these animals on days 5 to 15 compared to the nonhypoxic controls (Fig. 3). Increased megacaryocyte counts may increase the capacity for platelet production and explain the rebound thrombocytosis on days 13 to 15 (Fig. 2). Competition on common stem cells for erythropoiesis and thrombopoiesis during this phase could possibly also be the explanation for the decreased hematocrit levels found in the hypoxic animals on days 11 to 15 after birth compared to the control animals (Fig. 1).

A transitory coagulopathy with platelet consumption could possibly explain the postnatal thrombocytopenia with rebound thrombocytosis found in newborn mice after intrauterine hypoxia. In three newborn human infants with thrombocytopenia and hematocrit levels above $65 \%$, Rivers (10) suggested a low-grade disseminated intravascular coagulation to be present. However, a recent and more extensive study on newborn infants with poly- cythemia, hyperviscosity, and thrombocytopenia did not show coagulation pattern consistent with activated coagulation (3).

A small plasma volume in relation to the raised erythrocyte volume after intrauterine hypoxia could cause a decreased platelet count of whole blood in presence of a normal platelet concentration in plasma. In the present investigation. the elevation of hematocrit levels after intrauterine hypoxia decreased from $20 \%$ to zero compared to nonhypoxic control animals (Fig. 1) during the first wk after birth. During the same time. the platelet counts were decreased 30 to $40 \%$ compared to the controls (Fig. 2). The low whole blood platelet counts after intrauterine hypoxia therefore cannot be explained from an increased erythrocyte volume.

In newborn human infants with intrauterine growth retardation. polycythemia and a transitory postnatal thrombocytopenia are major hematologic findings (8). Placental dysfunction with impaired fetal oxygenation may be the cause of these effects, which were experimentally reproduced in newborn mice after chronic intrauterine hypoxia in this study.

\section{CONCILUSION}

Growth retardation. polycythemia, and a transitory postnatal thrombocytopenia followed by increased bone marrow megacaryocyte concentrations and a rebound thrombocytosis occurred in the offspring of mice exposed to long-term hypoxia during pregnancy. The existence of common stem cells for erythropoiesis and megacaryopoiesis may explain the interdependency between red blood cell and thrombocyte production. During chronic fetal hypoxia hematopoietic cells may be shunted in direction of erythropoiesis at expense of thrombopoiesis.

\section{REFERIN(ISS ANI) NOTHS}

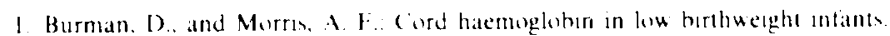
Arch. Dis (hild. 4l) $3 \times 2$ (1474).

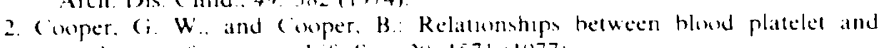

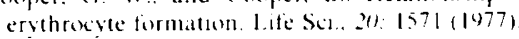

3. Henrikson. P.: Hepersocosity of the bloed and hatmostass in the newhorn infant. Acta Paediatr. Scand. os: 701 (1979).

4. Humbert. J. R. Abelorn. H. Hathawas. W. L... and Battagha. I ( . Polventhemat in small fir gestathonal age infants. J. Pediatr. $-5: 812(1969)$

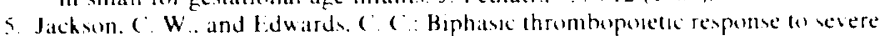
hypubaric hypoxia. Br. J Hatematol.. 3.5: 233 (1977).

b. 1.ichty. J. A. Fing, R. Y.. Bruns, P. D.. and Dyar. I... Studien on hathe horn at high altitude. Am. J. Dis. ( hild. "3: $060(1957)$.

7. Meberg. A.. Haga, P. and Halvorsen. S. Pre- and post natal serum ervihroporetun (LSf) levels in mice and rats. Response to hypoxia. Lxp. Hematod. (C openh.) (Suppl 6). 73 : 37974 )

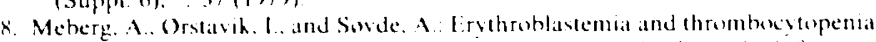
in small for gestational age infants. Relatwon to intraterine hypoxia. infectons and maternal smoking. Actal Patediats. Belg. 3/: 213 (1978).

4. Metcalf. I).. and Morre. M. A. S.: Haemopotete (ells pp. 195 211 (North. Holland Publishing (ompany. Amsterdam. 1971).

10. Rivers. R. P. A.: Coggulatwon changes associated with a high hatematecro in the newhorn infant. Acta Paediatr. Scand. of: $449(1975)$.

11. Shreiner. D). P.. and Levin. J.: The effects of hemorrhage. hyposta. and a preparation of erythropoietin on thromboporesis. J. 1.ab. ('lin. Med.. sis: y3t) (1976).

12. 1 want to thank (irethe Berg Pedersen for technical assistance.

13. Requests for reprints should be addressed to: Dr. Alf Meberg. Department of Pediatrics. Ulleval Hospital. Oslo. Norway.

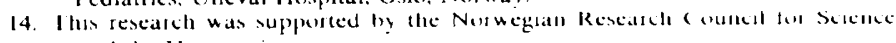
and the Humanties

15. Received for publication Nowember 7. 1979

16. Accepted for publication January 3. 1980. 